\section{Outcome Prediction to Erlotinib in Gastroesophageal Adenocarcinomas: Can We Improve Epidermal Growth Factor Receptor and Phospho-AKT Testing?}

To THE EDITOR: I read with great interest the recently published study by Dragovich et al, ${ }^{1}$ who reported on molecular biomarkers in a phase II trial of erlotinib for treatment of advanced gastroesophageal (GE) adenocarcinomas. Within a panel of candidate predictive markers of response, they analyzed epidermal growth factor receptor (EGFR) and phospho-AKT (pAKT) protein expression by immunohistochemistry (IHC) and EGFR gene status by fluorescence in situ hybridization (FISH). EGFR and pAKT expression were positive in $86 \%$ and $68 \%$ of tumor biopsies, respectively. Responses to erlotinib were observed in $9 \%$ of patients with gastroesophageal junction (GEJ) adenocarcinoma, and in $0 \%$ of gastric cancer patients. Given such modest response rates, and overall high frequency of EGFR- and pAKT-positive tumors, the authors concluded that assessment of these two biomarkers does not help to predict response. Furthermore, no EGFR amplification by FISH analysis was detected in samples from patients responding to erlotinib, and thus FISH does not appear to provide an adequate tool to predict response either. In an attempt to interpret the lack of predictive value yielded by IHC and FISH, this study actually does raise a few points concerning relevant methodologic details that warrant further discussion.

In the Methods section of their report, the authors state that the slides were read as positive or negative for EGFR and pAKT expression, and that positive staining was classified as weak $(1+)$ or strong $(2+)$. However, they do not mention the percentage of positive tumor cells they considered as the cutoff point to define EGFR and pAKT positivity, nor do they mention the breakdown of tumors deemed as $1+$ or $2+$ in terms of staining intensity. Nevertheless, the score they adopted implies that a positive result is defined by any staining intensity (from $1+$ to $2+$ ) in a fraction of tumor cells above a certain cutoff. Whether differences in scoring criteria would also translate into differences of outcome prediction is unknown. To define the level of expression of EGFR and pAKT by IHC, other researchers ${ }^{2}$ reported a combined score based on the percentage of positive tumor cells multiplied by the staining intensity. Importantly, this score significantly correlated to clinical outcome after gefitinib therapy in non-small-cell lung cancer (NSCLC). ${ }^{2}$ Although caution remains mandatory in extrapolating data from the non-small-cell lung cancer experience to GE cancers, it might be the case that a combined score expands the scope of IHC to detect any differential EGFR or pAKT expression level on a continuous scale. Whereas a positive result in the study by Cappuzzo et $\mathrm{al}^{2}$ might truly represent a high level of protein expression being detected, in most of the reported studies, including the one by Dragovich et al, ${ }^{1}$ a positive IHC result corresponds to any level of protein expression being detected. Therefore, even if they may have classified the staining intensities into two subcategories, weak or strong, these differences in scoring criteria might explain why their score failed to detect any correlation between protein expression and outcome.

From a technical point of view, some pitfalls might specifically complicate the assessment of pAKT by IHC. Janmaat et $\mathrm{al}^{3}$ recently found it particularly difficult to interpret pAKT staining in GEJ adenocarcinomas because the surrounding healthy tissues do express pAKT to some extent. Indeed, these investigators were able to assess pAKT status in only eight GEJ samples with tumor-specific staining. ${ }^{3}$ As a result, they do not even recommend an evaluation of pAKT as a predictor of response, although in their series some correlations could be established between the staining incidence and the staining intensity of this biomarker, and clinical outcome after gefitinib therapy. ${ }^{3}$ One can argue whether similar difficulties might also have intervened in the study by Dragovich et al, ${ }^{1}$ thus further explaining why assessment of pAKT did not retain any strong predictive value in their study.

With regards to FISH analysis, the authors need to clarify how they scored EGFR gene status and how they came up with the definition of EGFR amplification. This is standard practice in most publications. Although increased EGFR gene copy number in GE cancers has been described in earlier investigations, ${ }^{4,5}$ the increased EGFR copy number itself would probably be too low to define it as a marker for gene amplification by FISH analysis. Nevertheless, similar to the experience with gefitinib ${ }^{2,6}$ or erlotinib ${ }^{7}$ in NSCLC, if we exclude EGFR amplification, there remains the possibility that other genetic aberrations, for instance chromosome 7 polysomy, would have to be considered because of their possible impact on outcome prediction to tyrosine kinase inhibitors.

\section{Nicola Personeni}

Gastrointestinal Oncology Unit, Gasthuisberg University Hospital, Leuven, Belgium

\section{AUTHOR'S DISCLOSURES OF POTENTIAL CONFLICTS OF INTEREST}

The author indicated no potential conflicts of interest.

\section{REFERENCES}

1. Dragovich T, McCoy S, Fenoglio-Preiser CM, et al: Phase II trial of erlotinib in gastroesophageal junction and gastric adenocarcinomas: SWOG 0127. J Clin Oncol 24:4922-4927, 2006

2. Cappuzzo F, Hirsch FR, Rossi E, et al: Epidermal growth factor receptor gene and protein and gefitinib sensitivity in non-small-cell lung cancer. J Natl Cancer Inst 97:643-655, 2005

3. Janmaat ML, Gallegos-Ruiz MI, Rodriguez JA, et al: Predictive factors for outcome in a phase II study of gefitinib in second-line treatment of advanced esophageal cancer patients. J Clin Oncol 24:1612-1619, 2006

4. al-Kasspooles M, Moore JH, Orringer MB, et al: Amplification and overexpression of the EGFR and erbB-2 genes in human esophageal adenocarcinomas. Int J Cancer 54:213-219, 1993

5. Kimura M, Tsuda $H$, Morita $D$, et al: A proposal for diagnostically meaningful criteria to classify increased epidermal growth factor receptor and c-erbB-2 gene copy numbers in gastric carcinoma, based on correlation of fluorescence in situ hybridization and immunohistochemical measurements. Virchows Arch 445:255-262, 2004

6. Hirsch FR, Varella-Garcia M, McCoy J, et al: Increased epidermal growth factor receptor gene copy number detected by fluorescence in situ hybridization associates with increased sensitivity to gefitinib in patients with bronchioloalveolar carcinoma subtypes: A Southwest Oncology Group study. J Clin Oncol 23:6838-6845, 2005

7. Tsao MS, Sakurada A, Cutz JC, et al: Erlotinib in lung cancer: Molecular and clinical predictors of outcome. N Engl J Med 353:133-144, 2005

DOI: $10.1200 / J C O .2006 .09 .8137$ 